\title{
Effects of Chronic Marijuana Use on Brain Activity During Monetary Decision-Making
}

\author{
Jatin G Vaidya*', Robert I Block², Daniel S O'Leary', Laura B Ponto ${ }^{3}$, Mohamed M Ghoneim² and \\ Antoine Bechara ${ }^{4,5}$ \\ 'Department of Psychiatry, University of lowa Hospitals \& Clinics, lowa City, IA, USA; ${ }^{2}$ Department of Anesthesia, University of lowa Hospitals \& \\ Clinics, lowa City, IA, USA; ${ }^{3}$ PET Center, Department of Radiology, University of lowa Hospitals \& Clinics, lowa City, IA, USA; ${ }^{4}$ Brain and Creativity \\ Institute, University of Southern California, Los Angeles, CA, USA; ${ }^{5}$ Department of Psychiatry, McGill University, Montreal, Canada
}

Marijuana (MJ) acutely acts on cannabinoid receptors that are found in numerous brain regions, including those involved in reward processing and decision-making. However, it remains unclear how long-term, chronic MJ use alters reward-based decision-making. In the present study, using [ $\left.{ }^{15} \mathrm{O}\right]$ water PET imaging, we measured brain activity in chronic MJ users, who underwent monitored abstinence from MJ for approximately $24 \mathrm{~h}$ before imaging, and control participants, while they took part in the lowa Gambling Task (IGT), a monetary decision making task that strongly relies on the ventromedial prefrontal cortex (vmPFC). During PET imaging, participants took part in the standard and a variant version of the IGT as well as a control task. Chronic MJ users performed equally well on the standard IGT, but significantly worse than controls on the variant IGT. Chronic MJ users and control subjects showed increased regional cerebral blood flow (rCBF) in the vmPFC on both versions of the IGT compared to the control task. In the two-group comparison, chronic MJ users showed significantly greater rCBF than controls in the VmPFC on the standard IGT and greater activity in the cerebellum on both versions of the IGT. Furthermore, duration of use, but not age of first use, was associated with greater activity in the vmPFC. Thus, chronic MJ users tend to strongly recruit neural circuitry involved in decision-making and reward processing (vmPFC), and probabilistic learning (cerebellum) when performing the IGT.

Neuropsychopharmacology (2012) 37, 6I 8-629; doi:I0.1038/npp.201।.227; published online 28 September 20। I

Keywords: marijuana; decision-making; ventromedial prefrontal cortex; chronic use; cerebellum; PET

\section{INTRODUCTION}

Marijuana (MJ) is the most commonly used illicit drug in the Unites States (National Survey on Drug Use and Health, 2008). The acute effects of MJ are thought to involve the action of delta-9-tetra-hydrocannabinol (THC) on $\mathrm{CB}_{1}$ receptors located principally on axons and nerve terminals (Mechoulam, 1970; Matsuda et al, 1990; Iversen, 2003) in a number of regions, including the frontal cortex, hippocampus, and cerebellum (Iversen, 2003; Eggan and Lewis, 2007). However, the long-term impact of chronic MJ use on the brain remains poorly understood, especially with regard to more complex decision-making processes. In the present study, using $\left[{ }^{15} \mathrm{O}\right]$ water PET imaging, we measured brain activity in a large sample of chronic MJ users and controls

*Correspondence: Dr JG Vaidya, W278 GH, Department of Psychiatry, University of lowa Hospitals and Clinics, 200 Hawkins Dr., lowa City, IA 52242, USA, Tel: + 319356 7916, Fax: + 3193538656 ,

E-mail: jatin-vaidya@uiowa.edu

Received 20 January 2011; revised 18 August 2011; accepted 19 August 2011 while participants performed the Iowa Gambling Task (IGT) (Bechara et al, 1994), a commonly used measure of complex monetary decision-making. In addition to group comparisons, correlational analyses were conducted to investigate the association of two key variables - age of first MJ use and duration of MJ use - with brain activity.

Chronic MJ users, tested after brief abstinence periods, generally perform poorly on the IGT (Fridberg et al, 2010; Wesley et al, 2011; Whitlow et al, 2004). One study found that performance was poor even after a 25-day period of abstinence (Bolla et al, 2005). In addition, performance on the IGT is inversely correlated with recent MJ consumption (Hermann et al, 2009; but see Quednow et al, 2007). The ventromedial prefrontal cortex (vmPFC) is thought to have a critical role in decision-making and performance on the IGT (Bechara et al, 1994, 2000). This region has been implicated in emotional and motivational processes, and may have a critical role in decision-making by integrating physiological signals associated with emotional states (Damasio, 1994; Bechara et al, 2000) and reward value information (Kable and Glimcher, 2007; O'Doherty, 2004). Thus, given the evidence for poor performance on the IGT, 
it is possible that MJ use modifies the functionality of the vmPFC. To date, only one neuroimaging study has examined brain activity during monetary decision-making in comparison to an appropriate decision-making control condition in chronic MJ users (Bolla et al, 2005). In this study, using $\left[{ }^{15} \mathrm{O}\right]$ water PET, the investigators showed that chronic MJ users showed lower activity in the vmPFC as well as reduced activity in areas of the parietal lobe and cerebellum. However, studies that have utilized other purportedly frontally mediated tasks have reported increased activity in the frontal lobe regions in chronic MJ users who underwent limited (6-36 h; Kanayama et al, 2004) or no abstention (Gruber \& Yurgelun-Todd, 2005) from MJ before imaging.

Thus, although there is some evidence that chronic MJ use disrupts decision-making, the effect of MJ on vmPFC function is not well characterized. To help address this issue, we measured brain activity using $\left[{ }^{15} \mathrm{O}\right]$ water PET imaging while participants performed the IGT in a large sample of control subjects and chronic MJ users who underwent a period of approximately $24 \mathrm{~h}$ of monitored abstinence from MJ before imaging. In addition to group comparisons, analyses were conducted to examine the association between the duration of $\mathrm{MJ}$ use and brain activity. Examining duration of MJ use is important because if greater MJ use progressively leads to changes in brain function, then duration of use should have a direct association with brain activity. Furthermore, because early MJ use may modify the normal maturation of the vmPFC, a second and related goal was to examine the effects of age of first use on brain function.

Another goal of the present study was to better characterize the decision-making deficit and associated functional brain activity by incorporating a variant version of the IGT. The disadvantageous decks on the standard IGT are associated with large immediate rewards, but even greater later punishments; thus, poor performance on the standard IGT may simply reflect an insensitivity to punishments rather than more complex decision-making. In order to tease apart these various explanations, Bechara et al, (2000) developed a variant IGT in which the advantageous decks are associated with large initial punishments, but even greater rewards. Thus, if chronic MJ users are simply insensitive to punishments, then they should perform poorly on the standard but not variant IGT. On the other hand, if chronic MJ users perform poorly on the standard and variant IGT and show significant differences in vmPFC activity on both versions of the task, this would provide further evidence for the possible long-term effects of chronic MJ use on vmPFC-mediated decision-making function.

\section{PATIENTS AND METHODS}

\section{Participants}

Participants were frequent MJ users and non-using control subjects taking part in a larger project examining MJ use and brain functioning. The MJ users had been using MJ for at least 3 years and at a rate of 5 or more times weekly on average for the last 2 years or more. Controls had not used MJ on more than 6 days in their lives or any other non-prescribed psychoactive drugs except alcohol on more than 5 days in their lives. All subjects were at least 18 years old, right-handed, and had adequate vision and hearing to perform the tasks. Exclusion criteria included history of dependence on alcohol or any illicit drugs other than MJ according to the Computerized Diagnostic Interview Schedule for DSM-IV (CDIS-IV) (Robins et al, 1999), positive urine screening tests for illicit drugs other than MJ, history of schizophrenia, history of a manic episode or current depression, history of mental retardation or brain disease unrelated to drug use, severe obesity (in light of scanner size limitations), and current use of prescribed psychotropic drugs or other drugs or medical conditions that might affect neuroimaging or cognitive testing. We selectively recruited MJ users whose use of illicit drugs other than MJ was as limited as feasible. Drug use history was obtained using the Addiction Severity Index (McLellan et al, 1992) and locally developed instruments. Subjects were recruited by advertisements and paid $\$ 620.00$ plus travel reimbursements for completing all study procedures, including the two overnight hospitalizations, adjusted up or down by $1 \%$ of their fictitious gains or losses on the IGT. The maximum possible actual adjustment based on IGT performance was $\$ 35.40$ ( $1 \%$ of a fictitious $\$ 3540$ ). Subjects were informed in advance of this adjustment based on IGT performance.

The experiment was conducted with the understanding and consent of each subject, following approval of the experimental protocol by the University of Iowa Institutional Review Board for the use of human subjects. The initial sample of participants consisted of 50 chronic MJ users and 38 controls. However, because of technical issues on one or more tasks, complete imaging data on all three tasks were available on 46 chronic MJ users (28 males), who were on average 24.32 years old $(S D=3.85$; range $=19.76$ 37.68 ), and 34 controls (18 males), who were on average 24.72 years old $(S D=5.25 ;$ range $=19.28-36.36)$. The average initial age of first MJ use in the chronic MJ-using group was 16.43 years old $(S D=1.93$, range $=13.11-20.96)$. The average duration of use for this group was 7.90 years $(\mathrm{SD}=3.62$, range $=3.62-22.69)$.

\section{Procedure}

The results reported here are for the decision-making portion of a broader PET protocol that included other tasks not relevant to questions addressed in the current analysis. Subjects participated in two overnight hospitalizations during which they were continuously housed on an inpatient clinical research unit. Their presence was verified by nursing staff every $15 \mathrm{~min}$. A cognitive test session and PET scanning session were scheduled on the second days of the first and second hospitalizations, respectively. For MJ users (but not control subjects), the duration of abstinence from MJ before these sessions was constrained to be $24-29 \mathrm{~h}$ by having them smoke a MJ cigarette immediately following each hospital admission and urine screening for drugs of abuse. MJ users smoked a MJ cigarette provided by the National Institute on Drug Abuse, following a paced smoking procedure similar to that of Block et al, (1992). Each cigarette contained about $22 \mathrm{mg}$ THC. The cognitive test session included administration of the Quantitative and 
Expression subtests of the Iowa Tests of Educational Development (Hoover et al, 2003), along with other tests (data from which will be reported separately). Participants were instructed not to use illicit drugs other than MJ for 3 days before hospitalization. Recent abstinence from drugs of abuse other than MJ (ie, amphetamine, methamphetamine, cocaine, and morphine) was verified on the morning of the PET session and the preceding morning by urine screening (ie, competitive binding immunoassay using DrugCheck Drug Test Cup, Express Diagnostics) followed, if necessary, by confirmatory gas chromatography-mass spectrometry. Subjects were excluded if they tested positive for any other drug of abuse. Negative urine tests imply that acute effects of the drugs tested are no longer present. Chronic MJ users were also tested for cannabinoids in urine and excluded if they did not test positive.

Participants took part in two versions of the IGT as well as a third control task. The order of the control and experimental tasks were systematically varied such that the control task was performed either before or after the two experimental tasks. However, in keeping with previous studies that have used both the standard and variant IGT (eg, Bechara et al, 2002), all participants first took part in the standard IGT task, followed by the variant IGT.

The standard IGT (A'B'C'D') is the IGT described in detail by Bechara et al, (1994). Briefly, participants choose from one of four decks of cards over the course of 10 blocks of trials (10 trials per block). Two decks are advantageous (ie, they lead to long-term monetary gain) and two decks are disadvantageous (ie, they lead to overall monetary loss). Each card is associated with some reward and, on occasion, some cards are also associated with monetary loss. Although the rewards are high on the disadvantageous decks, the punishments are even greater. Thus, participants must learn through trial and error, which decks are advantageous and which are disadvantageous. On the variant version of the IGT (E'F'G'H'; Bechara et al, 2000), the basic setup is the same as the standard version. However, in the variant version each card choice is associated with some amount of monetary loss and, on occasion, some monetary gain. In this case, on the advantageous decks, although the punishments are initially higher, the eventual rewards outweigh the losses, leading to overall monetary gain. Finally, in the control task, participants simply choose a card from each deck in a pre-determined manner (A-B-C-D-A-B-C-D). Thus, no decision-making was involved, but participants were still required to respond with a button press as in the other conditions. Furthermore, the gains and losses were programmed such that the cards in each deck alternate between gain only and gain plus loss. The net gain is zero for every two consecutive cards in each deck. Decks A and B involve a $\$ 100$ gain on one card followed by a $\$ 100$ gain plus a $\$ 200$ loss on the next card; the pattern is the same for decks $\mathrm{C}$ and $\mathrm{D}$, but the gains and losses are half as large.

\section{Image Acquisition and Analysis}

Pet imaging. PET data were acquired using the $\left[{ }^{15} \mathrm{O}\right]$ water bolus injection method with a Siemens ECAT EXACT HR + scanner (Siemens Medical Solutions USA, Knoxville, TN) operated in the 2D mode. Participants were required to abstain from nicotine and caffeine beginning $1 \mathrm{~h}$ before PET imaging. Nicotine use earlier that day was permitted; caffeine use was discouraged, but not exclusionary. Three chronic MJ users used nicotine on the morning of the PET session (average number of minutes before imaging for these three subjects $=136.7 \mathrm{~min} ; \mathrm{SD}=15.3$ ). Three chronic MJ users used caffeine on the morning of the PET session (exact number of minutes of use before imaging for caffeine was not assessed). No control subjects used either caffeine or nicotine. After positioning the participant within the field-of-view of the scanner, transmission imaging for attenuation correction was performed using $\left[{ }^{68} \mathrm{Ge}\right]$ rod sources. A small dose (ie, 'scout scan' $=15 \mathrm{mCi}$ ) of $\left[{ }^{15} \mathrm{O}\right]$ water was administered by IV bolus injection to determine optimal timing for subsequent injections (Hurtig et al, 1994). The participant was instructed about the nature of the specific task to be performed (as described above) and reminded to stay as motionless as possible during image acquisition. Imaging ( $5 \mathrm{~s} /$ image frame) commenced at $\left[{ }^{15} \mathrm{O}\right]$ water injection $(45 \mathrm{mCi} /$ dose $)$, and was timed to occur approximately during blocks 2 through 5 of the IGT, and lasted for $100 \mathrm{~s}$. Data from the $40 \mathrm{~s}$ post-bolus transit were summed and iteratively reconstructed (2 iterations, 8 subsets; Gaussian 5.0 FWHM, zoom $=2.57,128 \times 128$ matrix, 63 slices) into activity images. Parametric images (ie, 'flow' images in terms of $\mathrm{ml} / \mathrm{min}$ per $100 \mathrm{ml}$ ) were calculated from the activity images and sampled arterial blood curves. Although fully quantitative (ie, flow images derived from arterial blood sampling) were available for a portion of the subjects, the following analyses used activity data normalized to the global mean value. Previous work at our institution has found consistent results when comparing normalized activity methods to fully quantitative imaging for analyses similar to what was done in the current investigation (Arndt et al, 1996; Block et al, 2000).

Structural imaging. MRI scans were obtained for each subject with a standard T1-weighted MP-RAGE sequence on a 3T Siemens (Erlangen, Germany) MAGNETOM Trio scanner $(\mathrm{TE}=2.50 \mathrm{~ms}, \mathrm{TR}=2530 \mathrm{~ms}$, flip angle $=10 \mathrm{de}-$ grees, $\mathrm{NEX}=2, \mathrm{FOV}=256 \mathrm{~mm}$, matrix $=256 \times 256 \mathrm{~mm}$, $1.5 \mathrm{~mm}$ slice thickness).

\section{Preprocessing and Statistical Analysis}

Image pre-processing was conducted using SPM5 unless otherwise noted. PET images were aligned to each subject's individual T1 image using AIR 3.0 (Woods, Grafton, Holmes, Cherry, \& Mazziotta, 1998). T1 images were warped to a Talairach template brain using a 12-point affine warping algorithm. The transformation matrix was then applied to each subject's PET image. The images were then smoothed using a $12 \mathrm{~mm}$ FWHM Gaussian kernel. PET data were masked using AFNI's (Cox, 1996) 3dAutomask algorithm and voxel values were normalized by dividing by the mean value for that PET scan. For the purposes of this investigation, regional cerebral blood flow ( $\mathrm{rCBF}$ ) will refer to the normalized $\mathrm{rCBF}$ described above.

The $t$-tests and regression analyses (using 3dRegAna) were carried out in AFNI. Two sets of paired $t$-tests for controls and chronic MJ users were computed (standard IGT vs. control IGT and variant IGT $v s$. control IGT). 
For the between-group $t$-test, we first created two sets of difference images for each subject (as in the paired $t$-tests) and used this difference image in the between-group analyses. The difference images were also used in the regression analyses. For the chronic MJ users in the regression analyses, analyses were conducted to characterize the association between duration of MJ use, age of first MJ use, and chronological age with rCBF. Duration of use was positively correlated with chronological age $[r(44)=0.87, p<0.001]$, although the association between age of first use and chronological age was not significant $[r(44)=-0.14, p=0.34]$. Given the strong correlation between duration of use and chronological age, associations between brain activity and these variables were expected to be highly convergent. Consequently, in order to characterize the nature of the associations, correlations were also computed between chronological age and $\mathrm{rCBF}$ in the control sample. To the degree that the correlations between chronological age and $\mathrm{rCBF}$ don't converge in the two groups, the effect of duration of use may be independent of age. For the whole-brain analyses, we used an uncorrected threshold of $p<0.005$ with an extent threshold of 150 contiguous voxels. However, because we had previous reasons for focusing on the vmPFC, we lowered the $p$-value to 0.05 for these analyses and reported as significant any cluster within the vmPFC that contained at least 150 contiguous voxels of significant results.

\section{RESULTS}

\section{Sample Characteristics}

Demographic and drug use history are summarized in Table 1. Both samples were equally well-educated. Intellectual functioning was assessed using Quantitative and Expression subtests of the Iowa Tests of Educational Development (Hoover et al, 2003). Performance on the Iowa Tests are strongly linked to IQ scores (Spinks et al, 2007). There were no significant differences between the control and chronic MJ groups on these tests. The proportion of males in the control (61\%) and chronic MJ using group (53\%) was also not significantly different (Fisher's exact test, $p=0.50$ ). As shown in Table 1 , the control group reported no regular MJ use (defined as 3 or more days of use per week on average) whereas the chronic MJ using group reported 6.26 years, on average. Given that duration of use, as reported in the Methods, was 7.90 years on average, these statistics indicate that regular MJ use was initiated roughly 1.64 years after initial use. Chronic MJ users reported using MJ almost every day within the past 30 days. Chronic MJ users were also asked to indicate how many times they used MJ in an average week during the last month. (One 'time' was defined as one smoking episode, regardless of quantity.) The mean was 11.1 times $(S D=5.0$, range $=4-21)$. Alcohol consumption was not significantly different between the two groups although there was a trend for the chronic MJ-using group to have been more regular users of alcohol (defined as drinking on 3 or more days per week on average) and to have consumed alcohol more frequently in the past 30 days. There were significantly more current cigarette smokers in the chronic MJ-using group (49\%) compared with the control group (3\%; Fisher's exact test, $p<0.001$ ), and although the cigarette use was fairly low in chronic MJ using group,they smoked significantly more number of cigarettes per day in the past year than control group subjects (Table 1). Although the chronic MJ users had experimented with other illicit drugs, based on frequency of use, MJ was clearly their drug of choice. Control group participants reported no use of any illicit drugs except MJ. The percentage of chronic MJ users who had used illicit drugs, except MJ between 10-99 times in their lives was generally low (Table 1) and no one had used any of these drugs more than 99 times. Furthermore, chronic MJ users reported very limited recent use of these drugs and their recent use differed significantly from control participants - who reported no such use-only for amphetamines. Mean number of days of use in the past 30 days was as follows: amphetamines $(\mathrm{M}=0.19 ; \mathrm{SD}=$ $0.58 ; t(44)=-2.20, p=0.03)$, cocaine $(\mathrm{M}=0.02 ; \mathrm{SD}=0.15$; $t(44)=-1.00, p=0.33)$, ecstasy $(\mathrm{M}=0.02 ; \mathrm{SD}=0.15 ; t(44)=$ $-1.00, p=0.33)$, other opiates or analgesics $(\mathrm{M}=0.03$; $\mathrm{SD}=0.17 ; t(44)=-1.35, p=0.18)$, other sedatives, hypnotics, and tranquilizers $(\mathrm{M}=0.11 ; \mathrm{SD}=0.53 ; t(44)=-1.40$, $p=0.17$ ) ( $t$-statistic is based on Satterthwaite approximation of degrees of freedom; drug use data for one subject was not available). No one reported use of barbiturates, hallucinogens, heroin, inhalants, or methadone in the past 30 days.

\section{Behavioral Results}

Performance on the IGT (number of cards chosen from the advantageous decks minus the number of cards chosen from the disadvantageous decks for each block of 10 trials averaged across all 10 blocks) for the control (mean $=3.10$, $\mathrm{SD}=3.72)$ and chronic $\mathrm{MJ}$-using $($ mean $=2.45, \mathrm{SD}=3.06)$ groups did not differ on the standard version (A'B'C'D') of the IGT $(t(76)=0.85, p=0.40)$. However, the difference in performance between control (mean $=6.38, \mathrm{SD}=3.24)$ and chronic $\mathrm{MJ}$ users (mean $=4.51, \mathrm{SD}=3.45$ ) was significant on the variant (E'F'G'H') task $(t(76)=2.44, p=0.02)$. As shown in Figure 1, performance was above zero for both groups on both versions of the IGT, indicating advantageous performance on the standard and variant tasks by the controls and chronic MJ users. In terms of total amount of money won or lost, the control group (mean $=\$ 445$, $\mathrm{SD}=\$ 1808$ ) and chronic MJ-users group (mean $=\$ 134$, $\mathrm{SD}=\$ 1572$ ) did not differ on the standard IGT $(t(76)=0.81, p=0.42)$. The difference between the control group's (mean $=\$ 1573, \mathrm{SD}=\$ 1251$ ) and chronic MJ users' (mean $=\$ 1041, \mathrm{SD}=\$ 1402$ ) earnings on the variant version of the IGT fell slightly short of significance $(t(76)=1.74$, $p=0.09)$. Finally, we also examined the association between age of first use of MJ and duration of MJ use with IGT performance. None of the correlations reached conventional levels of significance (all $p$-values $>0.05$ ).

\section{Neuroimaging Results}

Standard IGT (A'B'C'D'). Initial analyses were conducted on the standard version of the IGT to determine what regions were active on this task compared with the control task for each group separately. The top portions of Table 2 reflect the within-group comparisons and the bottom 
Table I Demographics and Drug Use History

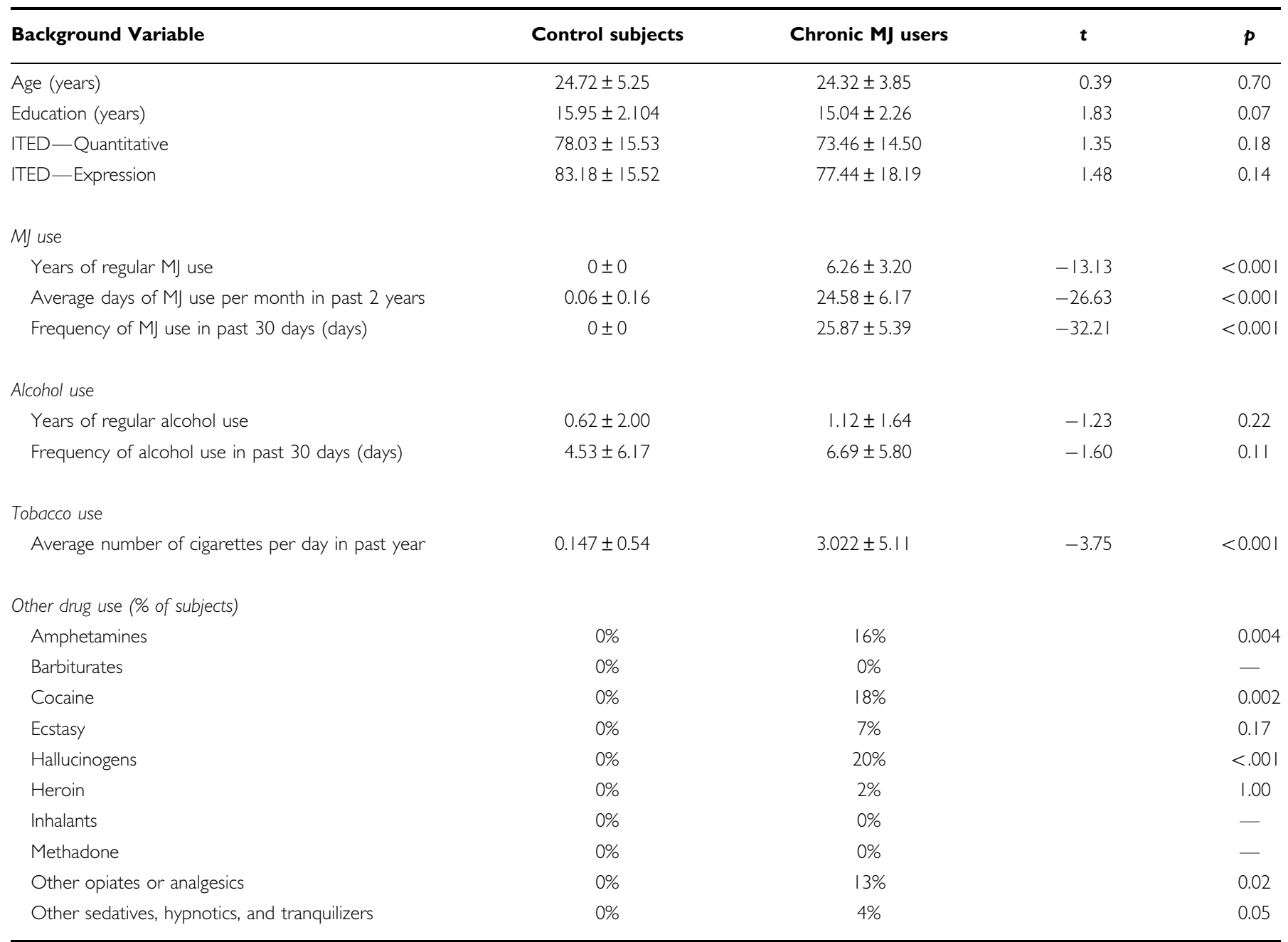

Note: ITED = lowa Tests of Educational Development (values represent normal curve equivalent scores). When variances differed across groups, $t$-values were based on Satterthwaite adjustment for unequal variances. Other drug use reflects percentage of subjects who reported using a given class of drugs between 10-99 times in their lifetime ( $p$-value is based on Fisher's exact test). No one used other drugs more than 99 times in their lifetime.

portions of the table represent regions that were significantly different between groups. As shown in Table 2, both groups recruited a similar set of brain regions during IGT task performance. Notably, both the control and chronic MJ-using groups showed a large cluster of activation in the vmPFC. Both groups also showed significant activity in the cerebellum. In the two group $t$-test, the chronic MJ users showed higher activity in the vmPFC and cerebellum compared with controls (see Figure 2). The control group on the other hand showed greater activity in the superior temporal gyrus in comparison with the chronic MJ-using group (see also Table 2 for a complete list of within-group and two-group comparisons).

The next set of analyses examined the correlation between brain activity on the standard IGT with age of first use and duration of use. Because duration of use is also correlated with chronological age, correlations were also computed to examine the association between this variable and normalized brain activity in each of the two groups separately to establish the specificity of the associations because of

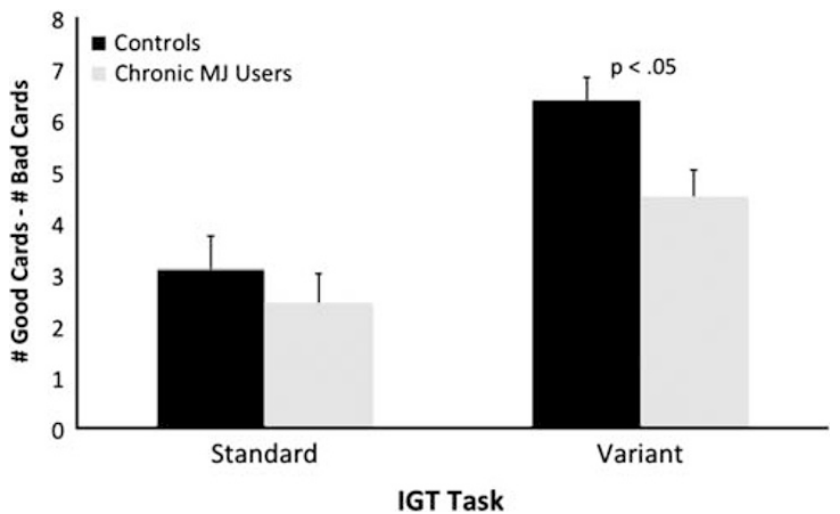

Figure I Performance on the standard (ie, $A^{\prime} B^{\prime} C^{\prime} D^{\prime}$ ) and variant (E'F'G'H') versions of the IGT. Performance represents the number of cards chosen from the advantageous decks minus the number of cards chosen from the disadvantageous decks for each block of 10 trials averaged across all 10 blocks. Higher scores indicate better performance. There were no group differences on the standard version of the task, but the difference on the variant was significant. Error bars = standard error of the mean. 
Table 2 Standard vs Control IGT Results

\begin{tabular}{|c|c|c|c|c|c|c|}
\hline \multirow[t]{2}{*}{ Region } & \multirow[t]{2}{*}{ L/R } & \multicolumn{3}{|c|}{ Talairach coordinates } & \multirow[t]{2}{*}{ Peak $t$} & \multirow{2}{*}{$\begin{array}{l}\text { Cluster } \\
\text { size }\end{array}$} \\
\hline & & $\mathbf{x}$ & $\mathbf{y}$ & $\mathbf{z}$ & & \\
\hline \multicolumn{7}{|l|}{ Control group } \\
\hline Anterior cingulate (BA 32) & M & 4 & 30 & 28 & 4.50 & 974 \\
\hline Inf. frontal gyrus (BA 47) & $\mathrm{R}$ & 36 & 16 & -8 & 4.09 & 715 \\
\hline vmPFC (BA 47) & $\mathbf{L}$ & -14 & 14 & -24 & 4.11 & 629 \\
\hline $\begin{array}{l}\text { Inf. frontal/sup. temporal gyrus } \\
\text { (BA 13) }\end{array}$ & $\mathrm{L}$ & -38 & 12 & -14 & 4.79 & $|13|$ \\
\hline $\begin{array}{l}\text { Cerebellum (inf. semi-lunar } \\
\text { lobule) }\end{array}$ & $\mathrm{L}$ & -40 & -64 & -42 & 6.10 & 3217 \\
\hline Cerebellum (tuber) & $\mathrm{R}$ & 48 & -66 & -30 & 5.19 & 341 \\
\hline Sup. frontal gyrus (BA 8) & $\mathrm{L}$ & -32 & 22 & 52 & -4.52 & 238 \\
\hline Supplementary motor area & $\mathrm{L}$ & -54 & -22 & 34 & -6.48 & 9848 \\
\hline Inf. parietal lobule (BA 40) & $\mathrm{R}$ & 50 & -24 & 22 & -3.90 & 282 \\
\hline Post-central gyrus (BA 5) & $\mathrm{R}$ & 18 & -42 & 66 & -4.60 & 212 \\
\hline Mid. temporal gyrus (BA 39) & $\mathrm{R}$ & 50 & -58 & 12 & -3.92 & 214 \\
\hline Sup. temporal gyrus (BA 39) & $\mathrm{L}$ & -60 & -62 & 22 & -4.60 & 405 \\
\hline \multicolumn{7}{|l|}{ Chronic MJ user group } \\
\hline Anterior cingulate (BA 32) & $\mathrm{R}$ & 2 & 40 & 16 & 3.42 & 227 \\
\hline vmPFC (BA 47) & $\mathbf{L - R}$ & 40 & 18 & -14 & 6.14 & 9432 \\
\hline Caudate & $\mathrm{R}$ & 10 & 0 & 18 & 4.10 & 161 \\
\hline Lentiform nucleus & $\mathrm{R}$ & 16 & -4 & -8 & 4.38 & 269 \\
\hline $\begin{array}{l}\text { Cerebellum (inf. semi-lunar } \\
\text { lobule) }\end{array}$ & $L-R$ & -38 & -66 & -36 & 9.94 & 9619 \\
\hline Supplementary motor area & $L-R$ & -22 & -22 & 66 & -7.08 & 17536 \\
\hline Post-central gyrus (BA 2) & $\mathrm{R}$ & 50 & -24 & 36 & -4.49 & 327 \\
\hline Cuneus (BA 19) & $\mathrm{R}$ & 6 & -84 & 32 & -3.50 & 335 \\
\hline \multicolumn{7}{|l|}{ Controls > CMJU } \\
\hline Sup. temporal gyrus (BA $2 \mathrm{I}$ ) & $\mathrm{L}$ & -38 & -6 & -18 & 3.83 & 152 \\
\hline \multicolumn{7}{|l|}{$\mathrm{CMJU}>$ Controls } \\
\hline vmPFC (BA 47) & $\mathbf{R}$ & 18 & 28 & -10 & 2.96 & 754 \\
\hline Cerebellar tonsil & $\mathrm{R}$ & 32 & -58 & -36 & 3.51 & 231 \\
\hline
\end{tabular}

Abbreviations: L, left; $M$, mesial; $R$, right.

Note: $N=34$ (comparison group); $N=46$ (Chronic MJ user group). Significance threshold set at $p<0.005$ ( $p<0.05$ for vmPFC group comparison); 150 contiguous voxels. In within-group analyses, positive $t$-values signify greater activity on the gambling task; negative $t$-values signify greater activity on the control task. A priori vmPFC findings are in bold.

duration of use. The duration and age of first use correlations are presented at the bottom of Table 3 . Associations with chronological age are presented at the top of Table 3. Duration of use was positively correlated with $\mathrm{rCBF}$ in a portion of the vmPFC and negatively correlated with $\mathrm{rCBF}$ in the dorsal anterior cingulate. Importantly, chronological age was also positively correlated with $\mathrm{rCBF}$ in the vmPFC in the chronic MJ users; however, age in the control group was not correlated with rCBF in the vmPFC. As chronic MJ users reported greater tobacco use, we conducted analyses to characterize the association between tobacco use and rCBF on the standard IGT (all tobacco use analyses are based on 45 subjects as tobacco use information was not available for one subject).

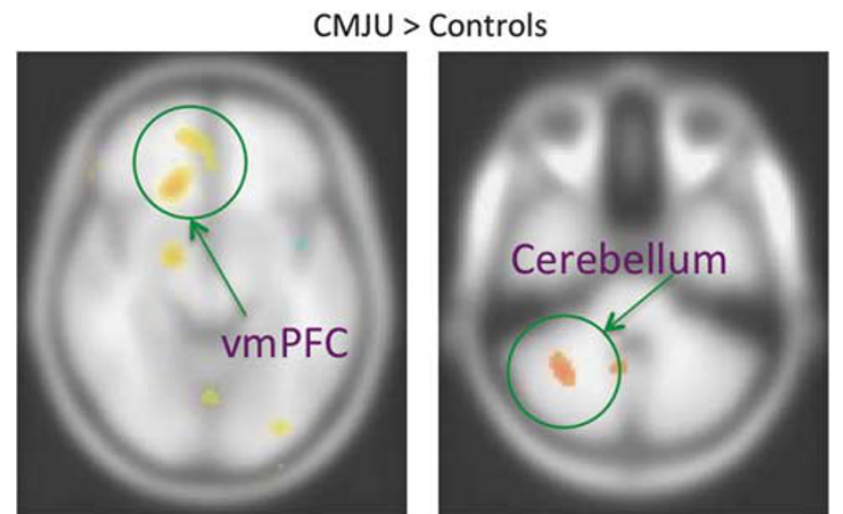

Figure 2 Differences between chronic $M J$ users and controls on the standard IGT. Results shown indicate that chronic MJ users showed greater activity in the vmPFC and in the cerebellum compared with control subjects.

Table 3 Associations with Activity on Standard IGT

\begin{tabular}{|c|c|c|c|c|c|c|}
\hline \multirow[t]{2}{*}{ Region } & \multirow[t]{2}{*}{$\mathbf{L} / \mathbf{R}$} & \multicolumn{3}{|c|}{ Talairach coordinates } & \multirow[t]{2}{*}{ Peak $r$} & \multirow{2}{*}{$\begin{array}{l}\text { Cluster } \\
\text { size }\end{array}$} \\
\hline & & $\mathbf{x}$ & $y$ & $\mathbf{z}$ & & \\
\hline \multicolumn{7}{|l|}{ Correlations with age: controls only } \\
\hline & & - & & & & \\
\hline \multicolumn{7}{|l|}{ Correlations with age: users only } \\
\hline vmPFC (BA I0) & M & -2 & 58 & 2 & 0.37 & 166 \\
\hline Cerebellum (cerebellar tonsil) & $\mathrm{R}$ & 14 & -48 & -34 & 0.48 & 155 \\
\hline \multicolumn{7}{|l|}{ Correlations with duration of use } \\
\hline vmPFC (BA I0) & M & -6 & 72 & 8 & 0.53 & 288 \\
\hline Anterior cingulate (BA 24) & M & 0 & 18 & 24 & -0.48 & $|5|$ \\
\hline \multicolumn{7}{|l|}{ Correlations with first use } \\
\hline Inf. frontal gyrus (BA |O/II) & $\mathrm{L}$ & -32 & 40 & 2 & 0.55 & 440 \\
\hline Precentral gyrus (BA 22) & L & -56 & 0 & 6 & 0.56 & 280 \\
\hline Precentral gyrus (BA 4) & $\mathrm{R}$ & 60 & -12 & 26 & 0.53 & 243 \\
\hline Precuneus (BA 7) & $\mathrm{R}$ & 24 & -64 & 36 & -0.58 & 245 \\
\hline
\end{tabular}

Abbreviations: L, left; $M$, mesial; $R$, right.

Note: $N=46$. Significance threshold set at $p<0.005$ ( $p<0.05$ for vmPFC); 150 contiguous voxels. Correlations are with activity during standard IGT v. control IGT. A priori vmPFC findings are in bold.

Tobacco use (average number of cigarettes smoked per day over the past year) was uncorrelated with both, duration of MJ use $(r(44)=0.01, p=0.96)$ and age of first MJ use $(r(44)=0.08, p=0.63)$. The average number of cigarettes smoked per day over the past year was negatively correlated $(r=-0.42)$ with $\mathrm{rCBF}$ in the vmPFC (BA $11 ; \mathrm{x}=-8, y=50$, $z=-12 ; 182$ contiguous voxels, $p<0.05)$. No other regions outside of the vMPFC were significantly correlated with tobacco use at $p<0.005$.

Variant IGT (E'F'G'H'). Results for the variant IGT are presented in Table 4. The top portions of table reflect the within-group comparisons and the bottom portions represent regions that were significantly different between 
Table 4 Variant vs Control IGT Results

\begin{tabular}{|c|c|c|c|c|c|c|}
\hline \multirow[t]{2}{*}{ Region } & \multirow[t]{2}{*}{$\mathbf{L} / \mathbf{R}$} & \multicolumn{3}{|c|}{ Talairach coordinates } & \multirow[t]{2}{*}{ Peak $t$} & \multirow[t]{2}{*}{$\begin{array}{c}\text { Cluster } \\
\text { size }\end{array}$} \\
\hline & & $\mathbf{x}$ & y & $\mathbf{z}$ & & \\
\hline \multicolumn{7}{|l|}{ Comparison group } \\
\hline vmPFC (BA 47) & L-R & 18 & 32 & -28 & 4.36 & 1834 \\
\hline Anterior cingulate (BA 32) & M & 4 & 24 & 38 & 5.27 & 761 \\
\hline Cerebellum (tonsil) & $\mathrm{R}$ & 20 & -48 & -38 & 4.95 & 281 \\
\hline Cerebellum (pyramid of vermis) & L & -4 & -74 & -28 & 4.96 & 2708 \\
\hline Inf. temporal gyrus (A 20) & $\mathrm{R}$ & 50 & -16 & -36 & -4.58 & 195 \\
\hline SMA (BA 3I) & $L-R$ & 0 & -20 & 46 & -3.83 & 427 \\
\hline Post-central gyrus (BA 3) & $\mathrm{L}$ & -44 & -24 & 58 & -4.70 & 244 \\
\hline Inf. parietal lobule (BA 40) & $\mathrm{R}$ & 52 & -26 & 24 & -4.94 & 469 \\
\hline Sup. temporal gyrus (BA 40) & $\mathrm{L}$ & -56 & -48 & 20 & -5.46 & 1921 \\
\hline Mid. temporal gyrus (BA 39) & $\mathrm{R}$ & 58 & -60 & 12 & -5.79 & 1017 \\
\hline \multicolumn{7}{|l|}{ MJ user group } \\
\hline Anterior PFC/vmPFC (BA I0) & $\mathbf{R}$ & 36 & 46 & 16 & 5.94 & 6139 \\
\hline Mid. frontal gyrus (BA 6) & $\mathrm{R}$ & 36 & 8 & 56 & 4.85 & 712 \\
\hline Cerebellum (inf. semi-lunar lobule) & $\mathrm{L}$ & -48 & -68 & -40 & 7.16 & 4236 \\
\hline Precuneus (BA 7) & $L-R$ & -14 & -80 & 50 & 5.37 & 1027 \\
\hline Sup. temporal gyrus (BA 38) & $\mathrm{R}$ & 34 & 2 & -46 & -5.20 & 1667 \\
\hline Inf. temporal gyrus (BA 20) & $\mathrm{L}$ & -40 & -2 & -36 & -6.81 & 3685 \\
\hline Insula (BA |3) & $L$ & -44 & -4 & 12 & -3.98 & 262 \\
\hline Sup. temporal gyrus (BA 39) & $\mathrm{L}$ & -52 & -60 & 18 & -6.16 & 2206 \\
\hline Mid. temporal gyrus (BA 39) & $\mathrm{R}$ & 50 & -66 & 10 & -4.88 & 437 \\
\hline \multicolumn{7}{|l|}{ Controls > CMJU } \\
\hline Mid. temporal gyrus (BA 38) & $\mathrm{L}$ & -40 & 2 & -36 & 4.21 & 399 \\
\hline \multicolumn{7}{|l|}{ CMJU > Controls } \\
\hline Insula & $\mathrm{L}$ & -34 & -20 & 16 & 4.01 & 270 \\
\hline
\end{tabular}

Abbreviations: $L$, left; $M$, mesial; $R$, right.

Note: $N=34$ (comparison group); $N=46$ (CMJU group). Significance threshold set at $p<0.005$ ( $p<0.05$ for vmPFC group comparison); I 50 contiguous voxels. In within-group analyses, positive $t$-values signify greater activity on the gambling task; negative $t$-values signify greater activity on the control task. A priori vmPFC findings are in bold.

groups. In general, the within-group results on the variant IGT were consistent with the standard IGT findings. Notably, both the controls and chronic MJ users showed increased $\mathrm{rCBF}$ in the vmPFC compared with the control task, although for the chronic MJ users, this activation was part of a large cluster of mesial to lateral PFC activation that had a peak in the middle frontal gyrus (BA 10). Also, as was the case on the standard IGT task, both groups showed strong activation in the cerebellum on the variant compared with control task. In fact, the two group comparisons only revealed two regions that showed significant differences in activation. The controls, compared with chronic MJ users, showed higher rCBF in the middle temporal gyrus (BA 38). Chronic MJ users showed higher $\mathrm{rCBF}$ in the left insula.

Although there was no difference in $\mathrm{rCBF}$ in the vmPFC between the two groups on the variant IGT task, there was a significant positive correlation between $\mathrm{rCBF}$ and duration of use (see Table 5). The correlation with chronological age and $\mathrm{rCBF}$ in the vmPFC in the chronic MJ users was also
Table 5 Associations with Activity on Variant IGT

\begin{tabular}{|c|c|c|c|c|c|c|}
\hline \multirow[t]{2}{*}{ Region } & \multirow[t]{2}{*}{ L/R } & \multicolumn{3}{|c|}{ Talairach coordinates } & \multirow[t]{2}{*}{ Peak $r$} & \multirow{2}{*}{$\begin{array}{l}\text { Cluster } \\
\text { size }\end{array}$} \\
\hline & & $\mathbf{x}$ & y & $\mathbf{z}$ & & \\
\hline \multicolumn{7}{|l|}{ Correlations with age: controls only } \\
\hline Cuneus (BA 19) & $\mathrm{R}$ & 10 & -82 & 30 & -0.54 & 198 \\
\hline Cuneus (BA |8) & $L$ & -14 & -88 & 12 & -0.51 & 190 \\
\hline \multicolumn{7}{|l|}{ Correlations with age: users only } \\
\hline Lingual gyrus (BA |8) & $\mathrm{R}$ & 10 & -66 & -2 & 0.53 & 236 \\
\hline $\begin{array}{l}\text { Mid. tem. gyrus/mid. occ. gyrus } \\
\text { (BA 19) }\end{array}$ & $\mathrm{R}$ & 36 & -64 & 16 & 0.58 & 163 \\
\hline vmPFC (BA I0) & $\mathbf{M}$ & -2 & 66 & 2 & 0.43 & 666 \\
\hline Sub-genual ACC (BA I0) & M & -14 & 34 & -10 & 0.37 & 329 \\
\hline \multicolumn{7}{|l|}{ Correlations with duration of use } \\
\hline Sup. temporal gyrus (BA 22) & $\mathrm{R}$ & 64 & -10 & 4 & -0.50 & 405 \\
\hline Lingual gyrus (BA |8) & $\mathrm{R}$ & 10 & -68 & 2 & 0.49 & 181 \\
\hline vmPFC (BA 10) & $\mathbf{M}$ & 0 & 66 & 4 & 0.37 & 464 \\
\hline Sub-genual ACC (BA I0) & M & -10 & 34 & -14 & 0.36 & 215 \\
\hline \multicolumn{7}{|l|}{ Correlations with first use } \\
\hline Inf. parietal lobule (BA 40) & $\mathrm{R}$ & 40 & -34 & 30 & -0.54 & 222 \\
\hline Precuneus (BA 19/39) & $\mathrm{R}$ & 30 & -62 & 38 & -0.54 & 208 \\
\hline Post-central gyrus (BA 43) & $\mathrm{R}$ & 62 & -16 & 20 & 0.47 & 154 \\
\hline Insula (BA |3) & $L$ & -46 & -4 & 14 & 0.54 & 152 \\
\hline
\end{tabular}

Note: $N=46$. Significance threshold set at $p<0.005$ ( $p<0.05$ for vmPFC); 150 contiguous voxels. Positive $t$-values signify positive correlations; negative $t$-values signify negative correlations. Correlations are with activity during variant IGT v. control IGT. A priori vmPFC findings are in bold.

significant, but the association with age in the controls was not. Age of first use was negatively correlated with rCBF in portions of the parietal lobe (post-central gyrus, inferior parietal lobule) and positively correlated with $\mathrm{rCBF}$ in the precuneus and insula. To better characterize this effect, we conducted a median split of the age of first use data and divided the two groups in half (early users and late users). The early users showed variant IGT-related activity in the insula that was significantly lower compared with the control task whereas the late users, like control subjects, did not differ in their activations in the two tasks. As with the standard IGT analyses, correlational analyses were computed to characterize the association between $\mathrm{rCBF}$ and tobacco use on the variant IGT. The average number of cigarettes smoked per day over the past year was negatively correlated $(r=-0.43)$ with $\mathrm{rCBF}$ in the vmPFC (BA 11; $\mathrm{x}=22, y=50, z=-10 ; 245$ contiguous voxels, $p<0.05)$. The average number of cigarettes smoked per day was also negatively correlated $(r=-0.49)$ with $\mathrm{rCBF}$ in the cerebellum (culmen; $\mathrm{x}=2, y=-48, \quad z=-22 ; 178$ contiguous voxels, $p<0.005)$.

\section{DISCUSSION}

The goal of the present study was to examine the effects of chronic MJ use on monetary decision-making and asso- 
ciated brain function. We utilized a large sample of chronic MJ users who abstained from MJ for approximately $24 \mathrm{~h}$ before imaging. Participants reported a clear preference for MJ, but also reported past use of other legal and illicit drugs. Although chronic MJ users and control participants activated a relatively similar set of regions on the decisionmaking task, chronic MJ users showed greater activation in regions implicated in emotional/monetary-decision making (vmpFC), the subjective experience of emotional states (insula), and probabilistic learning (cerebellum). Furthermore, duration of MJ use was positively correlated with activity in a number of regions involved in decisionmaking, including the vmPFC and anterior cingulate cortex. Earlier age of first use was not significantly correlated with activation in the vmPFC, but was positively correlated with activation in the insula. The significance of these results and limitations of the study are discussed below.

Although the acute effects of MJ on the brain are relatively well-characterized through animal studies (Iversen, 2003), the effect of long-term MJ use remains unclear (see, eg, Jager and Ramsey, 2008). This is a particularly salient issue given that $\mathrm{MJ}$ is (a) a widely used illicit drug, (b) some local and national governments have allowed the legal use of MJ for medical problems, and (c) there is an increasing push to legalize the recreational use of MJ. The present study compared brain activations between chronic MJ users and control subjects on a well-established monetary decision-making task - the IGT (Bechara et al, 1994). Furthermore, in order to better characterize the effects of chronic MJ use on brain function, analyses were conducted to examine how duration of chronic MJ use and age of first MJ use relate to the location and extent of brain activations. Although some studies have examined the effect of age of first use and duration of use on neural activation patterns, these analyses were typically of a post-hoc nature and consequently were based on relatively small sample sizes (eg, Tapert et al, 2007). In the present study, we recruited a large sample of chronic MJ users and control participants for the specific purpose of examining the effect of duration of use and age of first use on brain activations during decision-making.

Before turning to these correlations, we first discuss how the between group comparisons, both behaviorally and in terms of the functional neuroimaging results, converge with previous findings. The protocol in the present study used both standard (A'B'C'D') and variant (E'F'G'H') versions of the IGT. Unlike previous studies, we did not find differences in performance between control subjects and chronic MJ users on the standard IGT. However, the chronic MJ users did perform worse than control participants on the variant task. Importantly, although performance was worse, the chronic MJ users had a net score above zero, which indicates that they chose from the advantageous decks more than the disadvantageous decks, but not to the same degree as the control group. It is further noteworthy that participants always completed the standard IGT followed by the variant IGT. Thus, it is not clear if the poor performance on the variant IGT by the MJ group is directly related to task itself or if it is a function of the difficulty they had switching strategies from the standard IGT to the variant IGT. Arguing against this possibility is the fact that Solowij et al (2002) did not obtain a greater number of perseverative errors on the Wisconsin Card Sorting Test in MJ users compared with control subjects. Nevertheless, future studies which counter-balance the order of presentation would be better suited to examine this issue. Indeed, this is an important issue to explore because the variant IGT has punishments on each trial for all decks, but rewards on some of the decks. Performance on this task then may have real life implications in so far as many decisions involve negative repercussions, but differ in their rewarding properties. The lack of significant differences on the standard IGT was surprising given that several previous studies have reported significant group differences on this task (Bolla et al, 2005; Fridberg et al, 2010; Wesley et al, 2011; Whitlow et al, 2004). One potential reason for this difference may have been because the chronic MJ users in our sample had relatively high scores on measures of intellectual functioning. Furthermore, studies have varied in the imposed abstinence period ranging from approximately 12 (Fridberg et al, 2010; Wesley et al, 2011; Whitlow et al, 2004) to $24 \mathrm{~h}$ (as in the present study) to 25 days (Bolla et al, 2005). Also, although subjects' compensation was adjusted up or down by $1 \%$ of their fictitious gains or losses on the IGT in an effort to make the gambling situation more realistic (eg, Vadhan et al, 2009), the impact of this adjustment based on IGT performance may have been attenuated by its small size relative to the total amount that subjects were compensated for completing all study procedures. Additional studies are clearly needed to better characterize the full extent of the decision-making deficit in chronic MJ users, especially in relation to individuals who abuse other illicit substances, such as cocaine and amphetamine (eg, Bolla et al, 2003).

In terms of brain activation results, chronic MJ users and control participants engaged a number of common regions on the standard and variant tasks. For instance, both groups strongly activated the vmPFC and cerebellum on the IGT. However, the chronic MJ users showed significantly higher $\mathrm{rCBF}$ in these regions than control participants. In fact, chronic MJ users showed a bilateral increase in $\mathrm{rCBF}$ in the vmPFC whereas the effect was specific to the left vmPFC for the control group. The increased $\mathrm{rCBF}$ in the cerebellum is consistent with the findings of Bolla et al, (2005); however, we found higher $\mathrm{rCBF}$ in the vmPFC in the chronic MJ users whereas Bolla and colleagues reported lower activity in a portion of the orbitofrontal cortex overlapping with the vmPFC. Wesley et al, (2011), who asked subjects to abstain from MJ use after midnight before a functional MRI scan, also found lower activity in the ventromedial PFC in chronic MJ users to punishment feedback on an adapted version of the IGT. However, these investigators did not include a control condition that generated rewards and punishments. Consequently, the brain activation findings cannot be isolated to decision-making processes. One possible reason for the apparently discrepant results may be related to the fact that Bolla et al, (2005) had participants undergo a 25-day abstinence period before scanning. Participants in the present study underwent a 1-day period of abstinence. Thus, although we were able to establish that very recent MJ use did not contribute to differences in brain activations, there may have been residual effects of past MJ use that may not have been attenuated to the same degree as in the study by Bolla and colleagues. In addition, there were a number of 
differences in MJ user samples. Notably, the MJ users in the present study were more highly educated. Also, there were more cigarette smokers in the present study. Not withstanding the discrepancy with the earlier findings by Bolla et al, (2005), it is noteworthy that our results of greater vmPFC activity and lack of significant differences on the standard version of the IGT closely mirror the findings of an earlier study that compared control subjects to cocaine abusers on the IGT during PET imaging (Bolla et al, 2003).

Despite this inconsistency with an earlier study, we did find that duration of MJ use was positively correlated with activity in the vmPFC in the standard and variant versions of the IGT, lending internal consistency support to our findings. Interestingly, age of first use was not correlated with $\mathrm{rCBF}$ in this region. Thus, the findings in the present study indicate that greater MJ use was associated with higher activity in the vmPFC during monetary decisionmaking. The increase in $\mathrm{rCBF}$ in the vmPFC in the present study associated with greater $\mathrm{MJ}$ use may reflect a number of processes. The role of the vmPFC in decision-making is complex, but a number of studies suggest that this region has a central role in integrating affectively relevant information. From the perspective of the somatic marker hypothesis (Damasio, 1994), during the initial learning trials, various rewards and punishments elicit physiological changes. These physiological changes or somatic markers are then integrated within the vmPFC; effective integration can then provide a basis for appropriately choosing one deck over another (Bechara et al, 1999). Computational and neuroeconomic views on the function of the vmPFC also suggest that a key role of this region is to integrate information about rewards and punishments (Hare et al, 2008; Kable \& Glimcher, 2007). Thus, findings from this study may indicate that chronic MJ users show inefficiencies in reward-punishment integration processes. Indeed, given that chronic MJ users' decision-making was equivalent (standard IGT) or worse (variant IGT) than control subjects, it seems unlikely that the greater vmPFC activity reflects better reward-punishment integration. Given that the vmPFC is also implicated in more basic aspects of reward processing (as opposed to decision-making) (Kringelbach, 2005), another interpretation of the findings may be that the MJ users showed higher $\mathrm{rCBF}$ in this region because of greater sensitivity to rewards. But, undermining this possibility is the fact that all the activation findings were run against a control condition that included both punishments and rewards.

If future studies bear out the current findings, an important question to address is how chronic MJ use might alter the functionality of the vmPFC. Given the lack of significant findings with age of first use and $\mathrm{rCBF}$ in the vmPFC, the present results suggest that the effect may be relatively independent of age of first use. One possibility is that chronic MJ use leads to dysfunction in regions with high concentrations of cannabinoid receptors. These regions include the PFC, basal ganglia, hippocampus, and cerebellum. Indeed, several studies have shown that these very regions are disrupted in chronic MJ users (Iversen, 2003; Eggan and Lewis, 2007). Importantly, the PFC and especially the vmPFC also has a high density of dopaminergic receptors (Berger et al, 1991). Furthermore, there is some evidence that stimulation of cannabinoid receptors can alter dopaminergic transmission (Fox et al, 2002). In addition, a recent study found that CB1 and D2 receptors are clearly co-localized on PFC neurons and that activation of either the $\mathrm{CB} 1$ or $\mathrm{D} 2$ receptor suppress the release of GABA, the brain's primary inhibitory neurotransmitter (Chiu et al, 2010). Furthermore, long-term administration of THC to rats leads to an increase in dendrite length as well as dendritic branches in the medial PFC (Kolb et al, 2006). Taken together, these results suggest that long-term use of MJ may lead to heightened activation in vmPFC neurons. Although the findings do not specifically suggest that heightened vmPFC reflects an inefficient pattern of activation in this region, they establish a mechanism for how chronic MJ use may lead to increased activity in the PFC on a task that specifically targets this region.

Although the focus of this project was on the vmPFC, there were a number of significant findings that extended beyond this region. In particular, chronic MJ users showed higher $\mathrm{rCBF}$ in the cerebellum (standard IGT) and in the anterior insula (variant IGT), and age of first use was associated with greater activation in the posterior insula (variant IGT). Given that these results were not specifically hypothesized and not consistent across the different versions of the tasks, they should be interpreted with caution. Nonetheless, it is noteworthy that an increase in cerebellar activity in heavy MJ users was also reported by Bolla et al, (2005). These findings were consistent with the fact that the cerebellum is increasingly recognized as a region that is involved in a great deal more than simply motor coordination and motor learning (eg, Andreasen and Pierson, 2008; Schmahmann, 1991). Indeed, several studies have implicated the cerebellum in probabilistic reasoning and decision-making under uncertainty (Blackwood et al, 2004), possibly based on the representation of internal working models of behavior. Taking these findings in conjunction with the fact that chronic MJ users showed greater activity in the vmPFC, one interpretation of the data reported here is that chronic MJ users more strongly engage a set of distributed brain regions implicated in emotional decision making and integration of rewards and punishments (vmPFC) along with regions involved in action planning (cerebellum) to make advantageous decisions. Although the greater vmPFC and cerebellar activity is consistent with an inefficient processing view as mentioned earlier, it is important to note that higher activity in it of itself should not be taken to reflect a dysfunction in the brain. Given that the results presented here were inconsistent with the neuroimaging findings of Bolla et al, (2005), further research is needed to clarify the effects of chronic MJ use on the vmPFC, as well as other brain regions.

The analyses presented here also indicate that duration of MJ use was, in general, a much stronger predictor of activity in brain regions implicated in decision-making than age of first use. Importantly, our analyses indicate that chronological age does not appear to account for the association between duration of use and brain function as chronological age was not correlated with activity in these regions in the control group. The one exception to the absence of age of first use findings was in the insula. Although age of first use was positively correlated with activity in this region, followup analyses actually revealed a more complex association. Early MJ users showed higher activity on the control 
compared to the variant IGT whereas later MJ users showed equivalent activity on the variant and control task. Thus, the results indicate that early use was associated with a failure to recruit the insula on the variant IGT. Thus, it is not clear why early use of MJ would lead to lower activity in the insula on the variant IGT. This is a potentially important finding and should be investigated further, especially because of the role of the insula in awareness of internal states (Craig, 2002, 2009) as well as the link between insulamediated feelings of craving and addiction to nicotine (Naqvi et al, 2007).

\section{LIMITATIONS AND CONCLUSIONS}

Although there are a number of important implications of the findings presented here, they should be interpreted within the context of several limitations. Although the results of this study are consistent with the notion that chronic MJ use modifies brain functioning associated with monetary decision-making, the design of the present study does not allow for a causal conclusion about the link between MJ use and brain function or decision-making. Altough the present study was intended to focus on chronic effects of MJ use, several alternative explanations of the findings cannot be excluded. The 1-day period of abstinence from $\mathrm{MJ}$ that we required provided us with the resources to test substantially more individuals than would have been feasible with prolonged abstinence. This abstinence period was chosen in an effort to jointly minimize the risks of putative long-lasting acute effects (Leirer et al, 1991) and abstinence effects (Haney et al, 1999a,b). However, neither can be excluded with certainty. Moreover, cannabinoids are extremely lipid soluble, accumulate in fatty tissues, and are then slowly released back into other body compartments (Ashton, 2001). Cannabinoids may remain detectable in urine of frequent MJ users for 4 weeks (Pope et al, 2001a) or longer. Theoretically, some effects of chronic MJ use on cognition and brain function might persist for a number of days following abstinence, but disappear after more prolonged abstinence periods (Pope et al, 2001b). Such short-term effects may not reflect frank MJ-induced neurotoxicity. Although release of cannabinoids from fatty tissues back into the systemic circulation may not sustain blood levels high enough to affect brain function for very long, it is possible that THC or its metabolites may continue to bind to receptors even after a 24 -h period of abstinence, ultimately leading to increased activity in certain brain regions like the vmPFC. Indeed, this may explain why the present study, which implemented a $24-\mathrm{h}$ abstinence, found increased vmPFC activity, and the study by Bolla et al, (2005), which utilized a 25-day period of abstinence, found evidence for reduced activity in chronic MJ users in this region. Studies that involve prolonged abstinence or that focus on former MJ users who have ceased to use the drug may still not eliminate the possibility of very protracted but non-permanent effects of certain kinds (Schuckit et al, 1994) and may have increased susceptibility to selection bias (Pope et al, 2001a). A single study cannot address all these possibilities. Converging results from studies using different approaches are needed to reach confident conclusions.
In addition, it is possible that participants who use MJ have a pre-existing propensity to over-recruit and underrecruit certain brain regions. Furthermore, although we found significant correlations between $\mathrm{rCBF}$ and duration of use, it is similarly possible that individuals who ultimately use MJ for a longer amount of time had distinct neural profiles before use. In addition, whereas the chronic MJ users had a clear preference for MJ compared to other drugs, some subjects reported use of other illicit drugs besides $\mathrm{MJ}$ in the past. Chronic MJ users also reported smoking cigarettes significantly more than control participants. In this regard, it is important to note that existing evidence suggests that moderate levels of tobacco use is unrelated to performance on the IGT (Businelle et al, 2008; Harmsen et al, 2006). Moreover, in the present study, MJ use and tobacco use were not significantly correlated with one another in the MJ group. In fact, tobacco use was negatively correlated with $\mathrm{rCBF}$ in the vmPFC, whereas duration of $\mathrm{MJ}$ use was positively correlated with $\mathrm{rCBF}$ in the vmPFC. Thus, tobacco use does not appear to account for the association between duration of MJ use and activity in the vmPFC on the IGT. Nevertheless, these results should be more broadly interpreted as being reflective of an MJusing lifestyle (which may include use of some other drugs) rather than MJ per se (Jager et al, 2010) because we cannot exclude the effect of other drug use on the results. Finally, the potential for acute effects and abstinence effects of caffeine and nicotine among habitual users of these drugs is also of concern in functional neuroimaging studies (Dager and Friedman, 2000; Field et al, 2003). We attempted to minimize such effects by imposing modest limitations on caffeine and nicotine use. Although such effects cannot be excluded with certainty, few subjects used caffeine or nicotine on the morning of the PET session, which may suggest that such effects were not a significant problem.

Despite these limitations, the results presented here provide further evidence that MJ may alter brain functioning. The findings from this study extend previous human neuroimaging findings that have focused more on purely cognitive processes by examining brain activation on a monetary decision-making task. We hope these findings spur further research to examine the effects of chronic MJ use on motivational processes and decision-making. Indeed, this line of research will have a critical role in forming scientifically based national drug policies.

\section{ACKNOWLEDGEMENTS}

The authors thank Eugene Zeien for assistance with image processing, Levent Bayman for statistical assistance, and Jordan Zuccarelli, Megan Lombardi, and Catherine. Fruehling-Wall for help with data collection. This study was funded by NIH grant 5R01DA019338 to R.I.B.

\section{DISCLOSURE}

The authors declare that, except for income received from primary employers, no financial support or compensation has been received from any individual or corporate entity over the past three years for research or professional service and there are no personal financial holdings that could be perceived as constituting a potential conflict of interest. 


\section{REFERENCES}

Andreasen NC, Pierson R (2008). The role of the cerebellum in schizophrenia. Biol Psychiatry 64: 81-88.

Arndt S, Cizadlo T, O'Leary D, Gold S, Andreasen NC (1996). Normalizing counts and cerebral blood flow intensity in functional imaging studies of the human brain. Neuroimage 3: 175-184.

Ashton CH (2001). Pharmacology and effects of cannabis: A brief review. Br J Psychiatry 178: 101-106.

Bechara A, Damasio AR, Damasio H, Anderson SW (1994). Insensitivity to future consequences following damage to human prefrontal cortex. Cognition 50: 7-15.

Bechara A, Damasio H, Damasio AR, Lee GP (1999). Different contributions of the human amygdala and ventromedial prefrontal cortex to decision-making. J Neurosci 19: 5473-5481.

Bechara A, Dolan S, Hindes A (2002). Decision-making and addiction (part II): Myopia for the future or hypersensitivity to reward? Neuropsychologia 40: 1690-1705.

Bechara A, Tranel D, Damasio H (2000). Characterization of the decision-making deficit of patients with ventromedial prefrontal cortex lesions. Brain 123( Pt 11): 2189-2202.

Berger B, Gaspar P, Verney C (1991). Dopaminergic innervation of the cerebral cortex: unexpected differences between rodents and primates. Trends Neurosci 14: 21-27.

Blackwood N, Ffytche D, Simmons A, Bentall R, Murray R, Howard $\mathrm{R}$ (2004). The cerebellum and decision making under uncertainty. Brain Res Cogn Brain Res 20: 46-53.

Block RI, Farinpour R, Braverman K (1992). Acute effects of marijuana on cognition: Relationships to chronic effects and smoking techniques. Pharmacol Biochem Behav 43: 907-917.

Block RI, O'Leary DS, Hichwa RD, Augustinack JC, Ponto LL, Ghoneim MM et al (2000). Cerebellar hypoactivity in frequent marijuana users. Neuroreport 11: 749-753.

Bolla KI, Eldreth DA, London ED, Kiehl KA, Mouratidis M, Contoreggi C et al (2003). Orbitofrontal cortex dysfunction in abstinent cocaine abusers performing a decision-making task. Neuroimage 19: 1085-1094.

Bolla KI, Eldreth DA, Matochik JA, Cadet JL (2005). Neural substrates of faulty decision-making in abstinent marijuana users. Neuroimage 26: 480-492.

Businelle MS, Apperson MR, Kendzor DE, Terlecki MA, Copeland AL (2008). The relative impact of nicotine dependence, other substance dependence, and gender on Bechara Gambling Task performance. Exp Clin Psychopharmacol 16: 513-520.

Chiu CQ, Puente N, Grandes P, Castillo PE (2010). Dopaminergic modulation of endocannabinoid-mediated plasticity at GABAergic synapses in the prefrontal cortex. J Neurosci 30: 7236-7248.

Cox RW (1996). AFNI: software for analysis and visualization of functional magnetic resonance neuroimages. Comput Biomed Res 29: 162-173.

Craig AD (2002). How do you feel? Interoception: the sense of the physiological condition of the body. Nat Rev Neurosci 3: 655-666.

Craig AD (2009). How do you feel-now? The anterior insula and human awareness. Nat Rev Neurosci 10: 59-70.

Dager SR, Friedman SD (2000). Brain imaging and the effects of caffeine and nicotine. Ann Med 32: 592-599.

Damasio AR (1994). Descartes' Error: Emotion, Reason, and the Human Brain. Grosset/Putnam: New York.

Eggan SM, Lewis DA (2007). Immunocytochemical distribution of the cannabinoid $\mathrm{CB} 1$ receptor in the primate neocortex: a regional and laminar analysis. Cereb Cortex 17: 175-191.

Field AS, Laurienti PJ, Yen YF, Burdette JH, Moody DM (2003). Dietary caffeine consumption and withdrawal: Confounding variables in quantitative cerebral perfusion studies? Radiology 227: 129-135.

Fox SH, Henry B, Hill M, Crossman A, Brotchie J (2002). Stimulation of cannabinoid receptors reduces levodopa-induced dyskinesia in the MPTP-lesioned nonhuman primate model of Parkinson's disease. Mov Disord 17: 1180-1187.

Fridberg DJ, Queller S, Ahn WY, Kim W, Bishara AJ, Busemeyer JR et al (2010). Cognitive mechanisms underlying risky decision-making in chronic cannabis users. J Math Psychol 54: 28-38.

Gruber SA, Yurgelun-Todd DA (2005). Neuroimaging of marijuana smokers during inhibitory processing: a pilot investigation. Brain Res Cogn Brain Res 23: 107-118.

Haney M, Ward AS, Comer SD, Foltin RW, Fischman MW (1999a). Abstinence symptoms following smoked marijuana in humans. Psychopharmacology 141: 395-404.

Haney M, Ward AS, Comer SD, Foltin RW, Fischman MW (1999b). Abstinence symptoms following oral THC administration to humans. Psychopharmacology 141: 385-394.

Hare TA, O'Doherty J, Camerer CF, Schultz W, Rangel A (2008). Dissociating the role of the orbitofrontal cortex and the striatum in the computation of goal values and prediction errors. J Neurosci 28: 5623-5630.

Harmsen H, Bischof G, Brooks A, Hohagen F, Rumpf HJ (2006). The relationship between impaired decision-making, sensation seeking and readiness to change in cigarette smokers. Addict Behav 31: 581-592.

Hermann D, Lemenager T, Gelbke J, Welzel H, Skopp G, Mann K (2009). Decision making of heavy cannabis users on the Iowa Gambling Task: Stronger association with THC of hair analysis than with personality traits of the Tridimensional Personality Questionnaire. Eur Addict Res 15: 94-98.

Hoover HD, Dunbar SB, Frisbie DA, Oberley KR, Ordman VL, Naylor RJ et al (2003). The Iowa Tests: Guide to Research and Development: Forms A and B: Levels 5-14. Riverside Publishing: Itasca, Illinois.

Hurtig RR, Hichwa RD, O'Leary DS, Boles Ponto LL, Narayana S, Watkins GL et al (1994). Effects of timing and duration of cognitive activation in [15O]water PET studies. J Cereb Blood Flow Metab 14: 423-430.

Iversen L (2003). Cannabis and the brain. Brain 126: 1252-1270.

Jager G, Block RI, Luijten M, Ramsey NF (2010). Cannabis use and memory brain function in adolescent boys: a cross-sectional multicenter functional magnetic resonance imaging study. J Am Acad Child Adolesc Psychiatry 49: 561-572, 572e561-563.

Jager G, Ramsey NF (2008). Long-term consequences of adolescent cannabis exposure on the development of cognition, brain structure and function: an overview of animal and human research. Curr Drug Abuse Rev 1: 114-123.

Kable JW, Glimcher PW (2007). The neural correlates of subjective value during intertemporal choice. Nat Neurosci 10: $1625-1633$.

Kanayama G, Rogowska J, Pope HG, Gruber SA, Yurgelun-Todd DA (2004). Spatial working memory in heavy cannabis users: a functional magnetic resonance imaging study. Psychopharmacology 176: 239-247.

Kolb B, Gorny G, Limebeer CL, Parker LA (2006). Chronic treatment with Delta-9-tetrahydrocannabinol alters the structure of neurons in the nucleus accumbens shell and medial prefrontal cortex of rats. Synapse 60: 429-436.

Kringelbach ML (2005). The human orbitofrontal cortex: linking reward to hedonic experience. Nat Rev Neurosci 6: 691-702.

Leirer VO, Yesavage JA, Morrow DG (1991). Marijuana carry-over effects on aircraft pilot performance. Aviat Space Environ Med 62: 221-227.

Matsuda LA, Lolait SJ, Brownstein MJ, Young AC, Bonner TI (1990). Structure of a cannabinoid receptor and functional expression of the cloned cDNA. Nature 346: 561-564.

McLellan AT, Kushner H, Metzger D, Peters R, Smith I, Grissom G et al (1992). The fifth edition of the addiction severity index. J Subst Abuse Treat 9: 199-213. 
Mechoulam R (1970). Marihuana chemistry. Science 168: 1159-1166.

Naqvi NH, Rudrauf D, Damasio H, Bechara A (2007). Damage to the insula disrupts addiction to cigarette smoking. Science 315: 531-534.

O'Doherty JP (2004). Reward representations and reward-related learning in the human brain: insights from neuroimaging. Curr Opin Neurobiol 14: 769-776.

Pope Jr HG, Gruber AJ, Yurgelun-Todd D (2001a). Residual neuropsychologic effects of cannabis. Curr Psychiatry Rep 3: 507-512.

Pope Jr HG, Gruber AJ, Hudson JI, Huestis MA, Yurgelun-Todd D (2001b). Neuropsychological performance in long-term cannabis users. Arch Gen Psychiatry 58: 909-915.

Quednow BB, Kuhn KU, Hoppe C, Westheide J, Maier W, Daum I et al (2007). Elevated impulsivity and impaired decision-making cognition in heavy users of MDMA ('Ecstasy'). Psychopharmacology 189: 517-530.

Robins LN, Cottler L, Bucholz K, Compton W (1999). Diagnostic Interview Schedule for DSM-IV. Department of Psychiatry, Washington University School of Medicine: St Louis, MO.

Schmahmann JD (1991). An emerging concept. The cerebellar contribution to higher function. Arch Neurol 48: 1178-1187.

Schuckit MA, Kosten T, Fischman MW (1994). Protracted abstinence syndromes in alcohol, opioids, and stimulants. In: Widiger TA, Frances AJ, Pincus HA, First MB, Ross R, Davis W (eds). DSM-IV Sourcebook (Volume 1). American Psychiatric Association: Washington, D.C., 145-157.
Solowij N, Stephens RS, Roffman RA, Babor T, Kadden R, Miller $\mathrm{M}$ et al (2002). Cognitive functioning of long-term heavy cannabis users seeking treatment. JAMA 287: 1123-1131.

Spinks R, Arndt S, Caspers K, Yucuis R, McKirgan LW, Pfalzgraf C et al (2007). School achievement strongly predicts midlife IQ. Intelligence 35: 563-567.

Substance Abuse and Mental Health Services Administration (2008). Results from the 2007 National Survey on Drug Use and Health (NSDUH): National Findings. Series H-34, DHHS Publication No SMA 08-4343. Office of Applied Studies, Rockville, MD.

Tapert SF, Schweinsburg AD, Drummond SP, Paulus MP, Brown SA, Yang TT et al (2007). Functional MRI of inhibitory processing in abstinent adolescent marijuana users. Psychopharmacology 194: 173-183.

Vadhan NP, Hart CL, Haney M, van Gorp WG, Foltin RW (2009). Decision-making in long-term cocaine users: Effects of a cash monetary contingency on Gambling task performance. Drug Alcohol Depend 102: 95-101.

Wesley MJ, Hanlon CA, Porrino LJ (2011). Poor decision-making by chronic marijuana users is associated with decreased functional responsiveness to negative consequences. Psychiatry Res 191: 51-59.

Whitlow CT, Liguori A, Livengood LB, Hart SL, Mussat-Whitlow BJ, Lamborn CM et al (2004). Long-term heavy marijuana users make costly decisions on a gambling task. Drug Alcohol Depend 76: 107-111.

Woods RP, Grafton ST, Holmes CJ, Cherry SR, Mazziotta JC (1998). Automated image registration: I. General methods and intrasubject, intramodality validation. J Comput Assist Tomogr 22: $139-115$. 